\title{
Optical coupling of two distant InAs/GaAs quantum dots by a photonic-crystal microcavity
}

\author{
E. Gallardo, ${ }^{1, *}$ L. J. Martínez, ${ }^{2}$ A. K. Nowak, ${ }^{1}$ D. Sarkar, ${ }^{1,3}$ H. P. van der Meulen, ${ }^{1}$ J. M. Calleja, ${ }^{1}$ C. Tejedor,,${ }^{1}$ I. Prieto, ${ }^{2}$ \\ D. Granados, ${ }^{2}$ A. G. Taboada, ${ }^{2}$ J. M. García, ${ }^{2}$ and P. A. Postigo ${ }^{2}$ \\ ${ }^{1}$ Departamento de Física de Materiales, Universidad Autónoma de Madrid, E-28049 Madrid, Spain \\ ${ }^{2}$ Instituto de Microelectrónica de Madrid, Centro Nacional de Microelectrónica, Consejo Superior de Investigaciones Científicas, \\ Isaac Newton 8, PTM Tres Cantos, E-28760 Madrid, Spain \\ ${ }^{3}$ Department of Physics and Astronomy, University of Sheffield, Sheffield S3 7RH, United Kingdom
}

(Received 19 April 2010; published 11 May 2010)

\begin{abstract}
Long distance $(1.4 \mu \mathrm{m})$ interaction of two different InAs/GaAs quantum dots in a photonic crystal microcavity is observed. Simultaneous coupling of both quantum dots to the cavity is demonstrated by Purcell effect measurements. Resonant optical excitation in the $p$ state of any of the quantum dots, results in an increase in the $s$-state emission of the other one. The cavity-mediated coupling can be controlled by varying the excitation intensity. These results represent an experimental step toward the realization of quantum logic operations using distant solid-state qubits.
\end{abstract}

DOI: 10.1103/PhysRevB.81.193301

PACS number(s): 78.67.Hc, 42.50.Ex, 78.67.De

Efficient quantum information applications require qubits with low decoherence rates, fast manipulation times, and easy scalability. ${ }^{1}$ These requirements are met by qubits based on electron spins or excitons in semiconductor quantum dots (QDs). Coupling of single semiconductor QD excitons to a microcavity confined electromagnetic mode has different advantages depending on the coupling strength. Weak coupling allows enhanced optical efficiency associated to the exciton decay time reduction by the Purcell effect. ${ }^{2}$ In the strong-coupling regime, the system presents entangled lightmatter states that can be used as building blocks for transmission of quantum information, ${ }^{3}$ qubit readout, ${ }^{4}$ production of entangled pairs by compensation of the natural exciton fine structure splitting, ${ }^{5}$ and lasing. ${ }^{6}$ Single QD-cavity coupling has been demonstrated in the past years, ${ }^{7-13}$ showing interesting cavity-quantum electrodynamics effects. The possibility of using two or more qubits coupled by a single optical microcavity is appealing for it can provide techniques for long distance, fast interactions between qubits. ${ }^{14-16} \mathrm{New}$ dynamical phenomena are expected in these systems, which are dependent on the relative energy scales of the coupling between qubits and between qubits and the cavity mode (CM). In randomly distributed QDs samples it is statistically difficult to have two or more QDs both spatially and spectrally coupled to a microcavity mode. Some approaches have been proposed to obtain this type of coupled system, ${ }^{17-19}$ which rely on the deterministic location of the QD in the cavity. ${ }^{12,20}$ Coupling of several QDs to a single cavity mode has been reported as the origin of lasing at very low threshold. ${ }^{21}$

In this Brief Report, we show that exciton states of two semiconductor quantum dots with large lateral separation interact through a microcavity confined optical mode. Individual and simultaneous coupling of the QDs to the CM is demonstrated by changes in photoluminescence (PL) emission intensity and spontaneous emission rate (Purcell effect) when the QD excitons are brought into resonance with the CM. Cavity-mediated inter-QD interaction is demonstrated by PL excitation (PLE) measurements, in which resonant excitation at the $p$ state of any of the QDs increases the $s$-state emission of the other one. The microcavity-mediated inter- action of the two QDs can be controlled by varying the excitation intensity, i.e., the photon number in the CM.

The sample consists of a layer of randomly distributed self-assembled InAs QDs grown by solid source molecularbeam epitaxy. Atomic force microscope images of the QDs before capping show a ringlike shape, ${ }^{22}$ which is not relevant for the present results. The QDs are located inside a 158-nmthick GaAs slab grown on top of a $500 \mathrm{~nm}$ thick AlGaAs sacrificial layer. The QD surface density is $7.5 \times 10^{9} \mathrm{~cm}^{-2}$. The QD height average is $2 \mathrm{~nm}$ and the average lateral size is $50 \mathrm{~nm}$. A photonic crystal (PC) triangular lattice of holes of $140 \mathrm{~nm}$ diameter with a lattice constant of $230 \mathrm{~nm}$ was patterned by e-beam lithography and dry etching. Air suspended membranes were realized by sacrificial etching of the underlying AlGaAs layer. The optical cavity is formed by a missing hole in the PC and a slight inward shift of its nearest neighbors truncated holes (calzone cavity), ${ }^{23}$ as shown in Fig. 1(a). The quality factor is around $Q=3000$. The lowestenergy cavity mode is split into two components with orthogonal linear polarizations separated by $\sim 3.2 \mathrm{meV}$. Hereafter they will be designated as $\mathrm{X}$ and $\mathrm{Y}$ modes ( $\mathrm{X}$ corresponds to the long cavity diagonal). PL and PLE spectra of single QDs were taken with a micro-PL setup with a 2- $\mu \mathrm{m}$-wide Gaussian spot of a Ti-sapphire continuous laser. The in-plane position of the spot could be varied by $14 \mathrm{~nm}$ steps. This allows locating the individual QD within the cavity, by scanning the microscope objective, with $\pm 150 \mathrm{~nm}$ uncertainty, corresponding to a $10 \%$ decrease in the maximum PL intensity. Time-resolved PL (TRPL) measurements were taken with a pulsed diode laser emitting at $866 \mathrm{~nm}$ with energy below the wetting layer emission $(\sim 852 \mathrm{~nm})$. The $\mathrm{QD}$ exciton detuning $\left(\Delta=E_{\mathrm{QD}}-E_{\mathrm{CM}}\right)$ from the cavity mode was varied either by changing the temperature or by controlled Xe thin-film deposition. ${ }^{24}$ The emission spectra in Fig. 1(b) show the exciton emission of two QDs (QD1 and QD2) and the CM of X polarization. We find that QD1 and QD2 are at $0.5 \pm 0.15 \mu \mathrm{m}$ and $0.9 \pm 0.15 \mu \mathrm{m}$, respectively, away from the CM maximum almost in opposite directions along the $\mathrm{Y}$ direction [Fig. 1(a)]. The interdot distance is $1.4 \pm 0.3 \mu \mathrm{m}$. Figure 1(c) shows the crossing of QD2 and $\mathrm{CM}$ energies for increasing temperature. The CM was previ- 


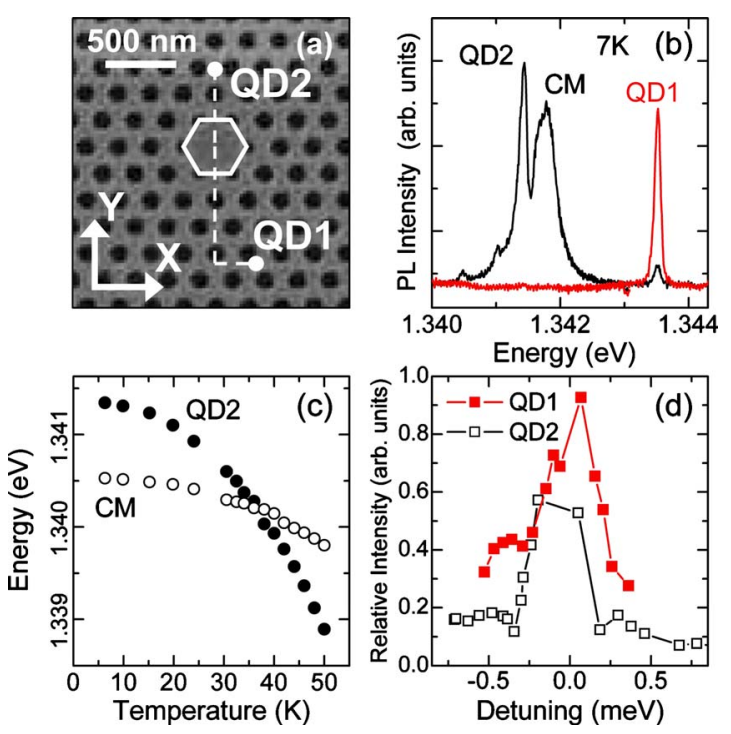

FIG. 1. (Color online) (a) Scanning electron microscopy image of the cavity structure with the approximate location of the QDs. (b) Photoluminescence spectra under nonresonant excitation showing the emission of $\mathrm{CM}$ and the QD1, QD2 excitons for $7 \mathrm{~K}$ for $\mathrm{X}$ (black line) and Y (red line) polarizations. (c) Energies of the QD2 and $\mathrm{CM}$ emission lines as a function of temperature. The $\mathrm{CM}$ was shifted to an energy higher than in (b) by Xe adsorption. (d) Emission intensity of QD1 (red) and QD2 (black) as a function of detuning for $\mathrm{X}$ polarization. The intensities are normalized to the total emission (the sum of the QD and the CM intensities).

ously shifted to lower energy by Xe adsorption to the sample surface. No measurable anticrossing is observed (neither in QD1), indicating weak coupling to the cavity. The experimental uncertainty of the energies sets an upper limit of several tens of microelectron volt for the QD-CM coupling strength. This is not surprising in view of the long distance from the QDs to the cavity center. However, recent theoretical work ${ }^{25,26}$ has shown that, under incoherent pumping, strong coupling can hold in the absence of a visible anticrossing due to decoherence-induced broadening of the lines. The individual QD coupling to the cavity is shown by temperature tuning of the QD excitons into resonance with the CM. Significant intensity increases are observed at zero detuning, as shown in Fig. 1(d) for both QDs. A stronger evidence of the QD coupling to the CM is shown in Fig. 2. The decay time of the QD spontaneous emission obtained by TRPL measurements is plotted against detuning for both QDs. The detuning scales are shifted to account for the energy difference between the emission of QD1 and QD2. In this way, points separated vertically correspond to the same measurement. A marked decrease in the emission time is observed for both QDs at small detunings (Purcell effect). In QD1 only positive detunings can be reached, as the temperature needed to change the detuning sign is too high for the PL to survive. In the detuning range between the vertical dashed lines both QDs are simultaneously coupled to the cavity mode. This roughly corresponds to the $\mathrm{CM}$ energy being intermediate between that of QD1 and QD2. The coupling strengths $g_{1,2}$ can be estimated from the total decay rate increase, which for large detunings $(\Delta \gg \kappa, g)$ is ${ }^{27}$

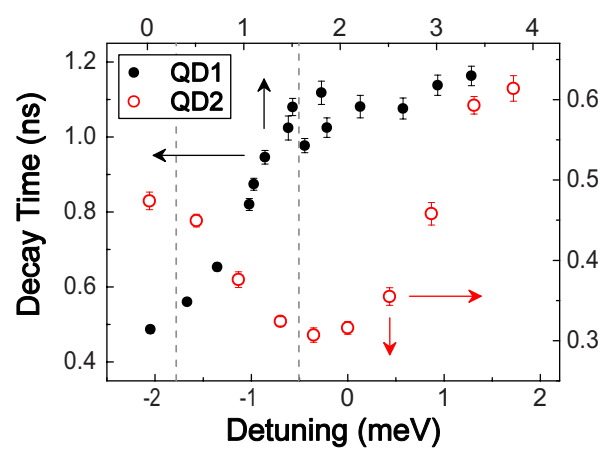

FIG. 2. (Color online) Decay time of the QD emission as a function of the detuning for both QDs. The detuning scales are shifted to account for the energy difference between the emission of QD1 and QD2. Up and left (down and right) scales correspond to QD1 (QD2). In the detuning range between the vertical dashed lines both QDs are simultaneously coupled to the CM.

$$
\tau_{1,2}^{-1}(\Delta)-\tau_{1,2}^{-1}(\infty)=\frac{4 g_{1,2}^{2}}{\kappa} \frac{\kappa^{2}}{\left(4 \Delta^{2}+\kappa^{2}\right)},
$$

where $\tau^{-1}(\infty)$ is the decay rate at large detunings and $\kappa$ is the spontaneous emission rate of the cavity photons. By fitting the data of Fig. 2 for $|\Delta|>0.5 \mathrm{meV}$ to Eq. (1) for both QDs we obtain $g_{1,2} \approx 30 \mu \mathrm{eV}$.

So far we have studied the individual coupling of the QDs to the CM. Now we will show how the cavity mediates an effective interaction between them. The emission intensity of the QDs and CM recorded at low power excitation $(0.2 \mathrm{~mW})$ is presented in Fig. 3 in a color scale as a function of excitation (vertical scale) and emission (horizontal scale) energies. The PLE maxima of both QDs are around $40 \mathrm{meV}$ above their emission energies. These maxima correspond to excited states, which we will simply call $p$ states. The $p$

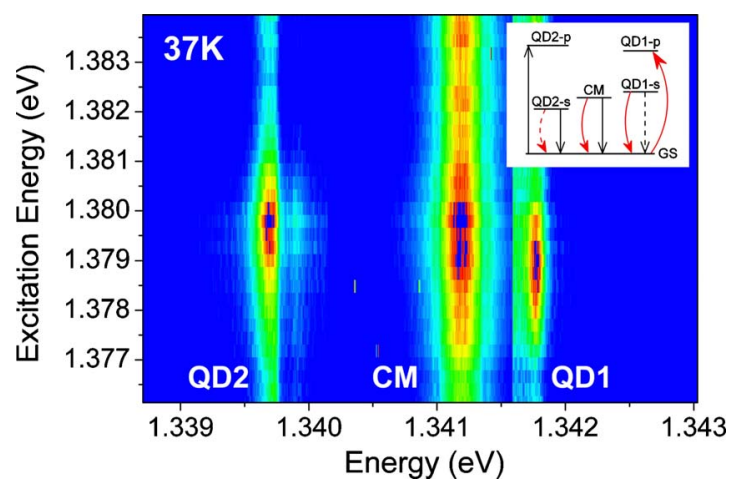

FIG. 3. (Color online) Emission intensity dependence of QD1, QD2, and CM (color scale) on excitation (vertical axis) and emission (horizontal axis) energies at $37 \mathrm{~K}$. The PLE maxima are around $37 \mathrm{meV}$ and $40 \mathrm{meV}$ above the emission for QD1 and QD2, respectively. The inset shows a diagram of the involved states and transitions. QD1,2-s, $p$ indicate the first and second exciton states of each QD. CM is the energy of a cavity photon. Red solid arrows show the excitation (up) and emission (down) processes of QD1. The dashed red arrow indicates the QD2 exciton transition induced by its coupling to QD1 through the cavity. Black arrows stand for the reciprocal case. 


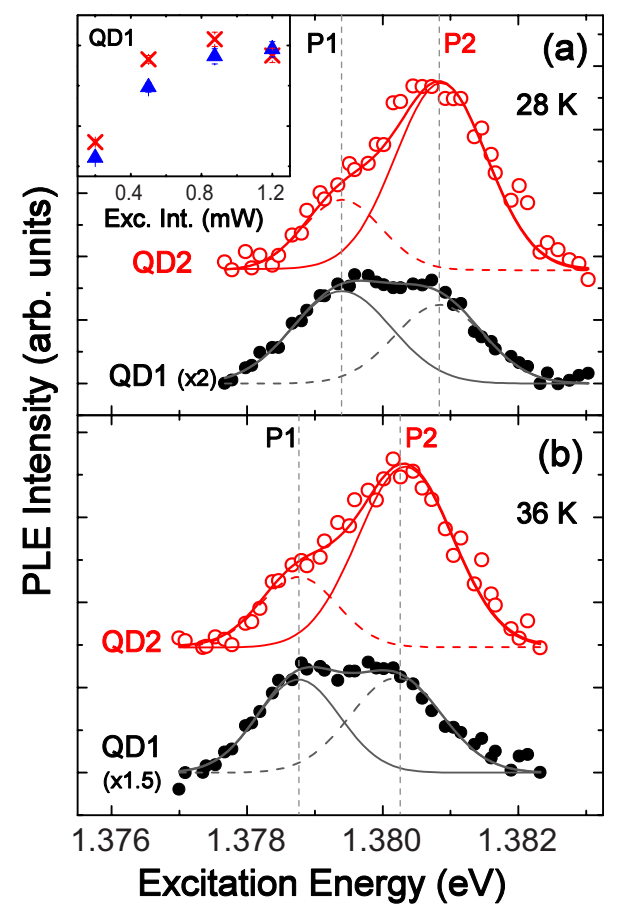

FIG. 4. (Color online) PLE spectra of QD1 and QD2 for X polarization at (a) $28 \mathrm{~K}$ and (b) $36 \mathrm{~K}$. The excitation intensity is 1.5 $\mathrm{mW}$ in both cases. $\mathrm{P} 1(\mathrm{P} 2)$ vertical line marks the $p$-state energy of QD1(QD2). All profiles are well fitted by two Gaussians. Dashed lines correspond to the PLE peak at the excitation energy of the $p$ state of the other QD. Inset: dependence of the QD1 PLE intensity on excitation intensity for intradot (red crosses) and interdot (blue triangles) excitation.

states of both QDs contribute to enhance the CM emission, as shown by the double maximum in the CM trace. The PLE spectra of the QDs (vertical cuts of Fig. 3) display small shoulders and asymmetries that evolve into new peaks at high excitation intensities. This is clearly seen in Fig. 4, where PLE spectra of QD1 (black solid circles) and QD2 (red open circles) taken with $1.5 \mathrm{~mW}$ excitation power, are shown for two different detunings. All spectra present a double-peak structure, which is well fitted by two Gaussians. The main maxima, also observed at low excitation intensity, correspond to excitation of the QDs at their respective $p$-state energies (P1, P2, marked by vertical dashed lines). In addition, both QDs show a new PLE peak (dashed Gaussian curves) at the excitation energy of the other QD. This "crossexcitation," schematically drawn in the inset of Fig. 3, proves the effective interaction between the two QDs. The amount of crossexcitation depends on the external pumping power (which determines the photon number in the cavity mode). The inset of Fig. 4 shows the evolution of the "intraQD" (red crosses) and "inter-QD" (blue triangles) contributions to the PLE intensity of QD1, obtained from the fits, as a function of the excitation intensity. A similar behavior is observed also in QD2. Both the intra-QD and the inter-QD contributions increase and saturate at high excitation intensity in a similar way.

The type of crossexcitation just described is not possible in independent QDs being 1.4 $\mu \mathrm{m}$ apart from each other. A rough estimate of the dipole-dipole interaction for $\mathrm{CdSe}$
QDs gives $1 \mathrm{meV}$ for an interdot distance $R=5 \mathrm{~nm} .{ }^{18}$ Assuming a similar value for the dipole matrix element for our InAs QDs, and considering the $1 / R^{3}$ decrease in the interaction, the direct dipole coupling would be in the range of $10^{-8} \mathrm{meV}$ for our dots. Consequently, coupling between the two QDs is only possible through their coherent interaction with the CM. The coupling would proceed in the following way: after resonant excitation at the $p$ state of one of the QDs, the electron-hole pair relaxes incoherently by phonon emission to the $s$ state of the same dot and becomes coherently coupled to the second QD by the CM. We can represent this process as $p_{1} \rightarrow s_{1} \Leftrightarrow \mathrm{CM} \Leftrightarrow s_{2}$, where $\rightarrow$ indicates incoherent decay and $\Leftrightarrow$ coherent coupling. A second channel, in which the excited state of the first QD decays incoherently into a cavity photon, exciting then the second QD $\left(p_{1} \rightarrow \mathrm{CM} \Leftrightarrow s_{2}\right)$, would lead to an incoherent interdot interaction. Although this second scenario cannot be completely ruled out, the trends observed in the inset of Fig. 4 indicate that the PL emission of a given QD increases sublinearly upon increasing the excitation intensity at either of the two QD $p$ states. If the interdot emission were due to direct photon injection from $\mathrm{CM}$, a linear trend would be expected, as the $\mathrm{CM}$ emission does not saturate. Consequently, the most probable interdot interaction mechanism involves the coherent coupling of the $s$ states by the cavity mode.

The physics of two qubits with no direct coupling but coupled to the same CM can be written, by means of a Schrieffer-Wolff transformation, in terms of an effective coupling between the qubits giving an effective Hamiltonian ${ }^{18}$

$$
\hat{H}=\frac{\hbar}{2} J\left(\hat{s}_{1}^{+} \hat{s}_{2}^{-}+\hat{s}_{1}^{-} \hat{s}_{2}^{+}\right), \quad J=\left(\frac{g_{1}^{2}}{\Delta_{1}}+\frac{g_{2}^{2}}{\Delta_{2}}\right),
$$

where $g_{i}$ is the coupling strength between qubit $i$ and the cavity mode, $\Delta_{i}$ is the detuning of qubit $i$ with respect to $\mathrm{CM}$, and $s_{i}^{ \pm}$are raising and lowering operators for qubit $i$. It describes the photon emission of one qubit into the cavity and subsequent absorption by the other qubit, giving rise to an effective cavity-mediated interqubit coupling. The highest $g$ values reported for InAs QDs are on the order of $0.1 \mathrm{meV}$ (Ref. 12) for QDs located near the cavity center. In our case $g_{i}$ are around $30 \mu \mathrm{eV}$, as determined from the Purcell measurements described above, so we can assume safely $g_{i} \ll \Delta_{i}$ for $0.5<\Delta_{i}<1.5 \mathrm{meV}$. As the observed QD emission energies are separated from the $\mathrm{CM}$ one by $\Omega_{i}=2 \sqrt{g_{i}^{2}+\left(\Delta_{i} / 2\right)^{2}}$, we have $\Omega_{i} \approx \Delta_{i}$, i.e., the observed emission energy differences with respect to the $\mathrm{CM}$ correspond approximately to the detuning values. From the detuning values $\left(\Delta_{1,2} \approx 1 \mathrm{meV}\right)$, and the $g_{1,2}$ values around $30 \mu \mathrm{eV}$, we can estimate $\Delta_{1,2} \approx 30 \mathrm{~g}$. Thus the effective coupling between the two quantum dots is $J \approx g_{1,2} / 15 \approx 2 \mu \mathrm{eV}$. This magnitude is much smaller than $\left|\Delta_{1}-\Delta_{2}\right|$ for typical values of the detunings. Therefore, population oscillations between the two qubits are expected to be slow enough to be easily detectable. ${ }^{18}$

In conclusion, we have demonstrated the effective coupling between two distant QDs mediated by the electric field of a confined cavity mode. Individual and simultaneous cou- 
pling is observed by the change in the spontaneous emission lifetime when tuning each QD in resonance with the CM. The cavity-mediated interaction is evidenced by the increased emission intensity of each QD upon resonant excitation at the $p$ state of the other one. The present results constitute an experimental step toward the realization of quantum logic operations using distant solid-state qubits.
The authors are indebted to E. Hu and R. Sabouni for helpful discussions. This work has been supported by research contracts of the Spanish Ministry of Education under Grants No. MAT2008-01555/NAN and Consolider No. CSD 2006-19 and Naninpho-QD under Grant No. TEC200806756-C03-01, and the Community of Madrid Grant CAM (Grant No. S2009/ESP-1503). *eva.gallardo@uam.es

${ }^{1}$ M. Feng et al., Phys. Rev. A 67, 014306 (2003).

${ }^{2}$ E. M. Purcell, Phys. Rev. 69, 681 (1946).

${ }^{3}$ S. J. van Enk et al., Phys. Rev. Lett. 78, 4293 (1997).

${ }^{4}$ A. Wallraff et al., Nature (London) 431, 162 (2004).

${ }^{5}$ R. Johne et al., Phys. Rev. B 79, 155317 (2009); P. K. Pathak et al., ibid. 79, 205416 (2009).

${ }^{6}$ M. Nomura et al., Nat. Phys. 6, 279 (2010).

${ }^{7}$ J. M. Gérard et al., Phys. Rev. Lett. 81, 1110 (1998).

${ }^{8}$ A. Kiraz et al., Appl. Phys. Lett. 78, 3932 (2001).

${ }^{9}$ J. P. Reithmaier et al., Nature (London) 432, 197 (2004).

${ }^{10}$ T. Yoshie et al., Nature (London) 432, 200 (2004).

${ }^{11}$ E. Peter et al., Phys. Rev. Lett. 95, 067401 (2005).

${ }^{12}$ A. Badolato et al., Science 308, 1158 (2005).

${ }^{13}$ M. Kaniber et al., Phys. Rev. B 77 161303(R) (2008).

${ }^{14}$ T. Pellizzari et al., Phys. Rev. Lett. 75, 3788 (1995).
${ }^{15}$ G. Burkard et al., Phys. Rev. B 74, 041307(R) (2006).

${ }^{16}$ E. del Valle et al., Phys. Rev. B 76, 235317 (2007).

${ }^{17}$ A. Imamoğlu et al., Phys. Rev. Lett. 83, 4204 (1999).

${ }^{18}$ O. Gywat et al., Phys. Rev. B 73, 125336 (2006).

${ }^{19}$ A. Imamoğlu et al., J. Appl. Phys. 101, 081602 (2007).

${ }^{20}$ A. Dousse et al., Appl. Phys. Lett. 94, 121102 (2009).

${ }^{21}$ S. Strauf et al., Phys. Rev. Lett. 96, 127404 (2006).

${ }^{22}$ D. Granados et al., Appl. Phys. Lett. 82, 2401 (2003).

${ }^{23}$ K. Hennessy et al., Appl. Phys. Lett. 89, 041118 (2006).

${ }^{24}$ S. Mosor et al., Appl. Phys. Lett. 87, 141105 (2005).

${ }^{25}$ F. P. Laussy et al., Phys. Rev. Lett. 101, 083601 (2008).

${ }^{26}$ A. Gonzalez-Tudela et al., Opt. Express 18, 7002 (2010).

${ }^{27}$ D. Englund et al., Phys. Rev. Lett. 95, 013904 (2005); M. Winger, Ph.D. dissertation, Eidgenössische Technische Hochschule (ETH), 2009. 